\title{
British Association for Paediatric Otorhinolaryngology (BAPO) abstracts from meeting on 8 October 2004
}

The Annual Meeting of BAPO was held on 8 October 2004 at Clifton Pavillion, Bristol.

\section{Hearing outcomes after surgery for cholesteatoma in children}

N K Chadha, D Owens, A Jardine

From the Royal United Hospital, Bath, UK.

\section{Introduction}

The management of cholesteatoma in childhood presents particular problems. Extensive disease is found more frequently in children compared to adults, and higher rates of residual and recurrent disease have been reported. There is as yet little consistency in the systems used to evaluate paediatric cholesteatoma, or the reporting of hearing outcomes. Comparisons between series are difficult, with meta-analysis of results impossible. The authors have identified very little research from the UK regarding hearing outcomes in paediatric cholesteatoma. The objective of this study was to determine the impact of type of surgical procedure and disease stage on the hearing status of children treated for cholesteatoma in our regional units.

\section{Method}

A retrospective case review of middle ear surgery for paediatric cholesteatoma was performed. All patients were treated in one of three regional units based in Bristol and Bath, with the first procedure performed between 1998 and 2000. Patients were only included if the following criteria were satisfied: (1) aged under 17 at time of operation, (2) first episode of cholesteatoma, and (3) the cholesteatoma was confirmed at surgery. Disease was staged by extent and ossicular state, and surgery classified as canal wall up (CWU) or canal wall down (CWD). Pure tone audiograms were measured pre-op and when disease-free, and reported as per the guidelines of the Committee on Hearing and Equilibrium (1995). Data was analysed to explore the statistical significance of initial stage and surgical technique on hearing outcome.

\section{Results}

Seventy-four patients were identified that met the inclusion criteria, with a mean age of 10.0 years (range 3-16). These comprised 51 boys (69 per cent) and 23 girls (31 per cent). Initial surgical technique was canal wall up (CWU) in 45 (61 per cent) and canal wall down (CWD) in 29 (39 per cent). 50 patients (68 per cent) presented with an intact stapes supra-structure and 24 patients (32 per cent) with an absent stapes supra-structure. Both the initial extent of disease and the initial ossicular state were found to be significantly better ( $p=0.002$ and $p=0.002$ respectively) in the group treated by the CWU technique. Mean air conduction hearing thresholds changed from
$47.6 \mathrm{~dB}$ pre-op to $44.1 \mathrm{~dB}$ post-op in the CWD group and from $35.8 \mathrm{~dB}$ pre-op to $29.8 \mathrm{~dB}$ post-op in the CWU group. Using a statistical model, it was found that after accounting for pre-operative hearing thresholds, CWU procedures resulted in a significantly better hearing improvement than CWD procedures for both air conduction thresholds $(p=0.002)$ and air-bone gaps $(p=0.016)$. No correlation was found between the initial extent of the disease and hearing outcome, but the presence of intact stapes supra-structure resulted in significantly better hearing outcome $(p=0.019)$.

\section{Conclusion}

This study found that children having CWD surgery for cholesteatoma had significantly worse initial disease than those having CWU surgery, signifying that surgeons were selecting the technique depending on the initial stage. Hearing outcomes were significantly better after CWU technique, or if the stapes supra-structure was initially present, but were not influenced by the initial stage.

\section{Paediatric cystic fibrosis patients receiving frequent} aminoglycoside therapy show evidence of reduced distortion product otoacoustic emissions prior to changes in pure tone audiometry

R Suryanarayanan ${ }^{*}, \mathrm{P}$ Hyman Taylor ${ }^{\dagger}, \mathrm{K} \operatorname{Tan}^{\dagger}, \mathrm{A}$ Smyth $^{\dagger}$, A Knox ${ }^{\dagger}$, D Stableforth ${ }^{\ddagger}$, and M Mulheran ${ }^{\S}$

From the Department of *ENT, Leicester Royal Infirmary; ${ }^{\dagger}$ Child Health \& Respiratory Medicine, Nottingham City Hospital; ${ }^{\ddagger}$ Respiratory Medicine, Birmingham Heartlands Hospital; ${ }^{\S}$ MRC Toxicology Unit, University of Leicester,UK.

\section{Introduction}

Cystic fibrosis patients require repeated high dosage therapy with aminoglycoside antibiotics. These drugs have a per course ototoxic risk of 5-10 per cent. Distortion Product Otoacoustic Emissions (DPOAEs) have previously been used to pick up subtle changes in cochlear outer hair cell (OHC) function in the absence of any significant changes in PTA. This study aimed to establish if any changes in DPOAEs could be seen in a group of paediatrics CF patients $(n=68)$ who had received repeated aminoglycoside therapy but were otherwise audiologically normal. These were compared with a group of similar aged paediatric controls $(n=43)$.

\section{Methods}

Standard PTAs were performed over 0.25 to $8 \mathrm{kHz}$ along with screening otoscopy and tympanometry. All subjects in both groups had DPOAEs measured at 2, 4 and $6 \mathrm{kHz}$ using an Otodynamics ILO 92. Stimulus parameters were: Intensity L1 = L2 dBSPL; f2/f1 $=1.22$. The $\mathrm{f} 2$ level 
generating a '(2f1-f2) DPOAE' of -10dBSPL provided an isoDP measure of sensitivity. Aminoglycoside exposure in the CF group was obtained by inspection of clinical notes: median number of courses 7; median dosage $38 \mathrm{~g}$; plasma levels within normal limits. Data were analysed by $t$-test.

\section{Results}

Both groups were comparable by age and sex distribution. All mean values of $-10 \mathrm{dBSPL}$ isoDP were elevated by 2-7 $\mathrm{dB}$ SPL in the CF group. This elevation reached significance $(p<0.001)$ at $4 \mathrm{kHz}$ in the left ear.

\section{Conclusions}

These changes provide some evidence of an early effect of aminoglycoside on cochlear OHC function preceding changes in the PTA. This may provide a further objective measure for the clinician for monitoring the onset of any ototoxic effects.

Interestingly in this group of paediatric $\mathrm{CF}$ patients, in spite of the repeated exposure, there was no evidence of frank aminoglycoside ototoxicity. This suggests that the risk of aminoglycoside ototoxicity in CF patients may be lower than that reported for non-CF patients.

\section{Reproducibility of nasal and exhaled nitric oxide measurements in children}

Stephen Lo, Hamid Daya, Nneka Eze, Wei Qian ${ }^{*}$, Blake Papsin ${ }^{*}$, Vito Forte

From the Department of Otolaryngology, St. George's Hospital Medical School, London SW17 0QT, UK; *Department of Otolaryngology, The Hospital for Sick Children, Toronto, Canada.

\section{Introduction}

Nitric Oxide (NO) is a molecular marker in exhaled air that can be used as a diagnostic tool as well as a way of monitoring the level of inflammation and oxidative stress in a variety of airway pathologies. In children in particular, airway NO measurement is an attractive tool of disease process monitoring as it is rapid and non-invasive. The aspiration technique for measuring nasal NO was recommended by the workshop sponsored by the American Thoracic Society in 1998. Although its reproducibility has been proven in adults very little data is available from children. The aim of the current study is to validate whether measurements of nasal and exhaled NO in normal healthy children using the aspiration technique are reproducible over a longitudinal period of time.

\section{Methods}

Both nasal and exhaled NO were measured in 9 healthy children over 5 consecutive days using a rapid-response chemiluminescent NO analyser. Statistical analysis was performed using ANOVA, with $p<0.05$ considered as significant.

\section{Results}

Of the 9 children there were 5 females and 4 males. The average age was 9.5 years (range: 6.6-13.4 years). The average inter-daily nasal nitric oxide output varied from 433.2 to $500.2 \mathrm{~nL} / \mathrm{min}$ and exhaled nitric oxide concentration ranged from 13.5 to $16.7 \mathrm{ppb}$ respectively. There was no significant difference among daily measurements on both nasal nitric oxide output (ANOVA $p=0.998)$ and on exhaled nitric oxide concentrations $(p=0.146)$.

\section{Conclusion}

In the current study, both nasal and exhaled nitric oxide measurements in children are reproducible over 5 days. Longitudinal measurements of nasal nitric oxide in children provide a valid mean of monitoring disease processes and may affect their treatments.

\section{Revision adenoidectomy: myth or reality?}

A Liapi, NO Turner, G Dhanasekar

From the Walsall Manor Hospital, Walsall, West Midlands WS2 9PS, UK.

\section{Introduction}

Adenoidectomy is one of the most commonly performed procedures in otolaryngological practice and various techniques have developed over the years. Blind curettage is the most popular technique currently used in the UK. Revision adenoidectomy for residual adenoids or genuine regrowth is gaining increasing awareness although the evidence in literature is scarce. In our paper we study the cases of revision adenoidectomies that were performed over the past eight years at a district general hospital mostly for recurrence of otological symptoms. We review the relevant literature and suggest more efficient methods of adenoidectomy under vision to prevent the recurrence of symptoms.

\section{Method}

The notes of 3231 children who underwent adenoidectomy between 1996 and 2003 were studied retrospectively. The standard procedure was blind curettage.

\section{Results}

A total of 53 required repeated operation for recurrence of symptoms (1.6 per cent). The mean age of the patients at first operation was 5.2 years. Of the 53 children that underwent revision adenoidectomy 42 were for treatment of glue ear, 6 for nasal symptoms and 5 for adenoidal infection.

\section{Conclusions}

Adenoidectomy performed without vision may be one of the reasons for recurrence of symptoms. Residual adenoids are acknowledged in literature as one of the complications of the traditional technique. We highlight the fact that the need for revision adenoidectomy is not uncommon and suggest that we should improve our technique by visualization of the PNS either by mirror or endoscope.

\section{Congenital nasal stenosis causing neonatal airway obstruction}

M Craig, G Morrison

From Guy's and St Thomas' Hospital, London, UK.

\section{Introduction}

Nasal obstruction is an uncommon but important cause of upper airway obstruction in neonates and infants. Neonates are obligate nasal breathers, so patency of the nasal airway is vital until the age of approximately three months. Recognised causes of nasal obstruction in this age group include neonatal and infantile rhinitis, choanal atresia and stenosis, piriform aperture stenosis, meningocoeles and gliomas, and primary nasal tumours. Other underrecognised causes such as traumatic deformation from passage through the birth canal and congenital nasal cavity stenosis are highlighted in this presentation. 


\section{Method}

Four recent cases of neonatal and infant nasal obstruction from nasal stenosis are presented.

\section{Results}

Four cases were seen in the first half of 2004. The common presentation was with stertor and recession, feeding difficulties, and difficulty in passing catheters through both nostrils, most commonly unilaterally. Patient ages were 5 days, 10 days, 6 weeks and 10 months. They all underwent full endoscopic upper airways examination under general anaesthesia, making the diagnosis and allowing endoscopic treatment. Case 1 was found to have unilateral nasal cartilaginous and bony obstruction with inferior turbinate protrusion, and underwent dilatation. Case 2 had been previously diagnosed with laryngomalacia and treated with aryepiglottoplasty but was found to have a bony mid-nasal obstruction which was treated by transnasal drilling and stent placement for six weeks. Case 3 had cartilaginous obstruction and inferior turbinate protrusion and was treated with nasal dilatation and outfracture of the inferior turbinates. Case 4 was found to have a septal cartilagenous spur and was treated by dilatation for outfracture. The choanae were normal in all four cases.

\section{Conclusion}

A neonate with significant signs of airway obstruction should be investigated by examination under anaesthesia of upper airways, including the nasal cavity, and microlaryngoscopy with bronchoscopy. For certain presentations, CT imaging may be appropriate. This presentation has demonstrated congenital nasal narrowing arising from the medial (septal) and lateral walls of the nose, and involving bone and/or cartilage.

The most important cause of congenital nasal obstruction is usually considered to be choanal atresia with an incidence of approximately 1 per 7000 live births. However, congenital nasal stenosis is another important and sometimes overlooked cause of infantile upper airways obstruction, and is surgically correctible. Based on our recent experience in a unit delivering 10,000 live births per annum, the incidence of significant nasal stenosis may be two or three times more common than choanal atresia.

\section{Congenital nasal pyriform aperture stenosis Konstantina} Tzifa, Michael Kuo, Ken Pearman

From the Department of Paediatric Otorhinolaryngology, Birmingham Children's Hospital, Birmingham, UK.

\section{Introduction}

Congenital nasal pyriform aperture stenosis is an unusual cause of upper airway obstruction in neonates and infants. Presentation may mimic choanal atresia and severity can vary from mild to significant airway obstruction. The efficient management of this condition requires establishment of a long-term adequate nasal airway. The purpose of this paper is to present our experience using a sublabial approach and enlargement of the nasal pyriform aperture by removing the nasal process of the maxilla, the narrowest part of the nasal airway.

\section{Method}

Seven patients are presented of which six underwent surgery with a sublabial approach with drilling of the nasal process of the maxilla or surgical dilatation. One patient was managed conservatively. All patients had a CT scan to diagnose this condition.

\section{Results}

Surgery is the definitive treatment with excellent longterm results. The six patients who had surgery required stents. A good nasal airway was established and maintained long term. Follow up has been between three months and five years. There has been no case of restenosis.

\section{Conclusion}

The sublabial approach provides enlargement of the nasal pyriform aperture with good access. Stents are essential to maintain a patent airway. While a conservative approach can be considered for mild cases, early surgical correction via the sublabial approach and removal of the nasal process of the maxilla is effective.

\section{Transnasal endoscopic treatment of bilateral choanal atresia using mitomycin $\mathbf{C}$ to avoid stenting}

James Tysome, Taran Tatla, William Grant

From the Department of Paediatric Otolaryngology, Chelsea and Westminster Hospital, 369 Fulham Road, London SW10 9NH, UK.

\section{Introduction}

Congenital bilateral choanal atresia is a rare malformation that causes airway obstruction in newborns. Many approaches have been used for treatment of choanal atresia including transpalatal, transnasal and transseptal. Most surgeons use post-operative stenting for several weeks to decrease rates of re-stenosis. Stents are associated with pain, local infections, granulation tissue formation and can affect nasal development. Mitomycin C is an aminoglycoside antibiotic widely used as a chemotherapeutic agent. Used topically it inhibits fibroblasts so decreasing granulation tissue. We describe our experience with a transnasal endoscopic technique for treatment of bilateral choanal atresia without postoperative stenting, using topical mitomycin $\mathrm{C}$ to prevent re-stenosis.

\section{Method}

Three patients aged 5 and 10 days and 4 years underwent transnasal endoscopic treatment of bilateral choanal atresia in October 2003 using 3 and $4 \mathrm{~mm}$ rigid nasal endoscopes. A drill was used for removal of abnormally widened vomerine septum posteriorly and pterygoid processes laterally. A $0.5 \mathrm{mg} / \mathrm{ml}$ solution of mitomycin C was applied to the choanae for 5 minutes. No stents were used. All patients underwent a second application of mitomycin $\mathrm{C}$ following debridement under anaesthesia at 3 weeks.

\section{Results}

All three patients have patent choanae at 9 month followup and have not required dilatations.

\section{Conclusion}

The use of mitomycin $\mathrm{C}$ following surgical repair of choanal atresia may improve patency and decrease the rates of dilatation and revision surgery so eliminating the need for post-operative stenting. 
Neonatal nasal microbiology and the factors that influence it: a prospective study

A Dasgupta, S Chattopadhaya, M Lyons, C Dutta, C Chowdhury

From the ENT Dept, Harold Wood Hospital, Gubbins Lane, Romford, Essex, UK.

\section{Introduction}

Bacterial colonisation of the nose is normal for humans. Babies are born essentially sterile with bacterial colonisation occurring shortly after birth. The factors that affect the time and extent of colonisation are unclear. We carried out a prospective study to investigate the nasal bacterial colonisation of neonates, both term and premature. We determined the onset of maximum colonisation, the factors influencing colonisation and whether there is a difference in the pattern of bacterial growth in the noses of pre-term infants.

\section{Methods}

Ethical approval and informed consent were obtained. Neonates, whether premature or term had nose swabs taken by aseptic technique by the authors. Neonates between zero and seven days were included in the study. Those over seven days old, neonates who received antibiotics, immunosuppressed neonates and those with clinical signs of infection were excluded form the study. The swabs were transported to the laboratory in Amies modified Stuarts transport medium. The swabs were cultured in blood agar in both aerobic and anaerobic conditions.

\section{Results}

51 neonates were studied. Of these 15 were normal deliveries and 34 were born by caesarean section and 2 had instrumental deliveries. Cultures were positive in 24 . Maximum colonisation occurred at day seven there appeared to be no colonisation at day zero. Growth of bacteria was found to be significantly higher in infants delivered vaginally when compared to caesarean section. The species of bacteria grown were similar to those reported in the literature for this age group. Premature infants showed equal levels of colonisation but staphylococcal species is more prevalent though not statistically significant.

\section{Conclusion}

Neonates born by vaginal delivery showed increased colonisation compared to other methods of delivery. Colonisation is maximal at day seven. Intervention (e.g. NG tube) in the neonate had no effect on bacterial growth. The bacteria grown on culture are those which have been documented in this age group. Staphylococcal species were more prevalent in the premature population although with small numbers of patients the difference was not statistically significant. The bacterial species grown in premature neonates were those which are more likely to have pathological effects e.g. CNS infections.

\section{Passive smoking, allergic rhinitis and nasal obstruction in children}

S De, J E Fenton, A S Jones, R W Clarke

From the ENT Department, Alder Hey Children's Hospital, Liverpool, L12 2AP, UK.

\section{Introduction}

Exposure to passive smoke has been implicated in a number of childhood disorders. There is evidence that children exposed to passive smoking have a poorer sense of smell and an increased risk of upper respiratory tract infections. It has also been shown that exposure to cigarette smoke affects nasal physiology. Allergic rhinitis is a very common childhood disorder and accounts for significant morbidity in this age group. We sought to assess the effect of passive smoking upon the symptom of nasal obstruction in children with and without allergic rhinitis.

\section{Methods}

This was a prospective study. Ethical approval was obtained from the regional children's ethics committee. Patients were recruited from the ear, nose and throat outpatient department. Each patient was asked to mark a score on a visual analogue scale for the degree of nasal obstruction. Exposure to passive smoking was determined subjectively using a parental questionnaire and objectively by measuring the urinary cotinine/creatinine ratio. Results were tabulated using Microsoft Excel and analysed with SPSS statistical software.

\section{Results}

Eighty-one children took part in the study. Thirty-one patients had a positive history of allergic rhinitis. There was a smoker in the household in almost half of the cases. There was a statistically significant association between a positive history of a smoker in the household and the cotinine/creatinine ratio. Nasal obstruction was significantly worse in children with a positive history of allergic rhinitis $(p<0.05)$. There was also a trend towards a higher degree of nasal obstruction in children without allergic rhinitis exposed to passive smoking when compared to those who weren't exposed to passive smoking. However, passive smoking did not appear to affect the obstruction score in children with allergic rhinitis.

\section{Conclusions}

Nasal obstruction is worse in children with allergic rhinitis than those without. There was a statistically significant association between subjective and objective measures of passive smoking. Passive smoking tends to increase the symptom of nasal obstruction in children without allergic rhinitis. It does not appear to have an effect on nasal obstruction in children with allergic rhinitis.

\section{A comparison of ultrasonic scalpel tonsillectomy with blunt dissection tonsillectomy in a paediatric age group - a single-blind randomised controlled trial}

WA Clement, S Loughran, M Oko, I Ganly, NK Geddes, D Young

From the Royal Hospital for Sick Children, Yorkhill, Glasgow, G3 8SJ, UK.

\section{Introduction}

Tonsillectomy is one of the most commonly performed otolaryngology procedures. Several different techniques for this procedure have been described, however, debate still remains as to whether any particular method gives superior results regarding post-operative pain. The ultrasonic scalpel was recently introduced as a new technique for tonsillectomy. This is a hand held device with an ultrasonically activated blade tip that vibrates at 55500 cycles per second. It acts on tissues by cutting by cavitational fragmentation and mechanical disruption and it also coagulates by coaptation. The aims of this study were to determine if there was a difference in the post- 
operative pain scores, and dietary intake, when comparing ultrasonic tonsillectomy (US) with blunt dissection tonsillectomy (BD).

\begin{abstract}
Methods
One hundred and twenty two children were recruited and randomised into blunt dissection and ultrasonic scalpel tonsillectomy groups. Two operators performed all tonsillectomies. Each technique was standardized. Bipolar diathermy was used for haemostasis when required. All tonsillectomy equipment used in the trial was disposable. Intra-operatively, operative time and blood loss were recorded. All patients stayed overnight for observation. Post-operative pain was recorded using an abbreviated 'faces' pain scale. Dietary intake was measured using a four point dietary scale. Pain and dietary intake were recorded on post-operative days one, three, five, seven and nine. All children were discharged with a one-week course of weight appropriately calculated paracetamol. Patients were contacted by telephone and reminded to complete their questionnaire forms two weeks post-operatively. The incidences of reactive and secondary haemorrhage in both groups were recorded, as were readmission rates for dehydration and pain.
\end{abstract}

\section{Results}

One hundred and twenty-two children were enrolled. Ninety-three $(76 \%)$ patients completed their dietary and pain scales. Post-operative pain was significantly greater in the US group overall $(p=0.0003)$ and on days one and three $(p=0.0466, p=0.0084)$. Dietary intake scores were significantly better in the US group on days one, five, seven and nine, however, dietary intake scores declined in both groups throughout the study. Blood loss was significantly greater in the BD group $(p<0.0001)$. There were no significant differences for all other observed outcomes.

\section{Conclusions}

US tonsillectomy confers advantages over BD tonsillectomy in decreased intra-operative blood loss but appears to cause significantly more post-operative pain overall and on days one and three. US tonsillectomy may be advantageous in children where tight control of blood loss is required but does appear to cause more pain.

\section{Success rates in removing nasal and aural foreign bodies in children}

C Webb, E Osman, M McCormick

From the Alder Hey Children's Hospital, Eaton Road, Liverpool, UK.

\section{Introduction}

Children are often seen in the casualty department having placed a wide variety of foreign bodies in their noses or ears. Often attempts by casualty doctors to remove these foreign bodies results in failure, a fractious child, and subsequent referral to the ENT department for removal. Removal in the ENT clinic was perceived to be made more difficult after a failed attempt in casualty. Failures in the ENT clinic necessitate general anaesthesia for successful removal. A retrospective analysis of all children presenting with nasal and aural foreign bodies to Alder Hey Children's Hospital was undertaken to evaluate this problem.

\section{Method}

One hundred and seventy-eight patients with nasal or aural foreign bodies were seen in the casualty department at Alder Hey in 2002. The casualty card for each patient was used to determine the site of the foreign body, whether a removal attempt was made, and the outcome of the attendance i.e. successful removal or referral to ENT clinic. Any patient referred to clinic had their case notes examined to see whether the foreign body was removed in clinic or under general anaesthesia. Data was analysed using an unpaired $t$-test.

\section{Results}

There were 71 nasal foreign bodies 55 were in the right nostril $(p<0.001, \mathrm{z}=3.9))$ over an $\mathrm{n}$ age range of 1 to 7 years. Sixteen were removed in casualty out of 56 attempts (28.6 per cent). Of the 40 failed attempts five were removed in ENT clinic (12.5 per cent) versus nine removals in the 15 patients who had no previous failed attempt at removal (60 per cent). This difference was highly significant $(p<0.01, \mathrm{z}=3.4704)$.

One hundred and seven patients had aural foreign bodies 72 were in the right ear $(p<0.01, \mathrm{z}=3.7)$. The patients ranged from 2 to 15 years old. Nineteen were removed in casualty out of 79 attempts (24 per cent). Eighty-eight patients were referred to the ENT clinic of which 60 had had a failed attempt. In these 60, 17 foreign bodies were removed successfully ( 28.3 per cent) and in the remaining 28 patients, 21 foreign bodies were removed successfully (75 per cent). This difference was highly significant $(p<0.001, \mathrm{z}=4.6483)$.

\section{Conclusion}

The results show that the likelihood of removal of paediatric nasal and aural foreign bodies in the ENT clinic decreases significantly if a previous attempt has failed. It is recommended that attempts at removal are undertaken by an experienced clinician, with appropriate equipment, good lighting and assistance from a nurse skilled in the proper handling of children with foreign bodies. The practicalities of this mean that all children with such foreign bodies are referred to the ENT department. Based on our results this would increase the workload due to foreign bodies by 19.6 per cent. Assuming we had seen all 178 patients and had a 60 per cent success rate in removing nasal foreign bodies and a 75 per cent success rate in removing aural foreign bodies we could have prevented 36 patients undergoing general anaesthesia for removal of their foreign bodies.

\section{The role of CT scans in retropharyngeal and parapharyngeal infections in children}

S Lo*, H Daya*, N Eze*, BC Papsin ${ }^{\dagger}$, A Zachariasova ${ }^{\dagger}, \mathrm{S}$ Laughlin ${ }^{\ddagger}, \mathrm{S}$ Blaser ${ }^{\ddagger}$

From the "Department of Otolaryngology, St. George's Hospital Medical School, London, UK; ${ }^{\dagger}$ Departments of Otolaryngology \& Radiology, Hospital for Sick Children, Toronto, Canada.

\section{Introduction}

Retropharyngeal (RP) and parapharyngeal (PP) infections in children are rare conditions but carry significant potential mortality and morbidity. Early diagnosis is necessary to prevent the development of complications. However, because of their variability in presenting symptoms and lack of physical signs, they present a diagnostic challenge to clinicians. The current study reviewed the Hospital of Sick Children, Toronto's experience of the diagnosis and management of RP and 
PP infections and examined the role of CT imaging in diagnosing the presence of an abscess.

\section{Methods}

A retrospective analysis of all patients diagnosed with RP and PP infections from 1987 to 1999 was performed. Demographic data, presenting symptoms, season of presentation, management and complications were reviewed. The CT scans of 21 patients who underwent surgical treatment were retrospectively examined by two neuroradiologists who were blinded to the patients' history and outcome. The sensitivity, specificity and predictive values for the specific features and overall assessment were calculated.

\section{Results}

Fifty four children were identified. There were 46 RP infections, 6 PP infections and 2 patients had both RP and PP infections. All patients were treated with parenteral antibiotics. Thirty-seven patients underwent surgical drainage and in 27 there was a positive finding of pus. The retrospectively assessed CT scans of the 21 patients who underwent surgery were found to have a sensitivity of 81 per cent in detecting an abscess by CT scan but the specificity was 57 per cent. There were four complications including mediastinitis, aspiration pneumonia, internal jugular vein thrombosis and common carotid artery aneurysm. All patients recovered but abscess recurred in five patients.

\section{Conclusion}

Not all patients with RP and PP abscesses require surgery. Whilst CT scans are helpful in diagnosing and assessing the extent of these infections they are not always accurate in detecting an abscess. A decision to drain an abscess should therefore not be made based solely on the CT findings.

\section{Adjuvant intravenous cidofovir in paediatric recurrent respiratory papillomatosis}

Marcel Geyer, Michelle Wyatt, Susanna Leighton

From the Department of Otolaryngology, Great Ormond Street Hospital for Sick Children, London WC1N 3JH, UK.

\section{Introduction}

Recurrent respiratory papillomatosis (RRP) is the commonest benign neoplasm of the paediatric larynx. The aetiology has been linked to epithelial infection by human papilloma virus (HPV) types 6 and 11 and may occur anywhere in the respiratory tract, but has a predilection for squamo-columnar junctions, and usually starts in the larynx. Presenting symptoms depend on the extent of the disease and range in severity, though there is a tendency towards slow, spontaneous disease regression at puberty. A definitive means of disease eradication is yet to be found. Treatment aims are an adequate airway and voice preservation. This is commonly achieved by laser debulking, with or without adjuvant topical or systemic therapies, facilitating disease remission while limiting morbidity. There is currently no established therapy for RRP with pulmonary spread. We report two paediatric cases of severe RRP with pulmonary spread treated by laser debulking and a course of systemic intravenous cidofovir.

\section{Method}

Two children with intractable upper respiratory tract and pulmonary papillomas were treated with a combination of https://doi.org/10.1258/002221505775010698 Published online by Cambridge University Pres laser debulking of upper airway disease and a course of intravenous cidofovir. Their response to therapy was measured by frequency of operation, signifying papilloma regrowth rate, over 6 and 10 years respectively, precidofovir therapy, and for one and a half years following the course.

\section{Results}

The first child underwent 147 procedures in just over 11 years to the present date (13.3 operations per year); the second patient had 97 operations in almost 8 years (14.1 operations per year). Prior to cidofovir they averaged an operation every 25 and 26 days respectively; post-cidofovir these rates were every 66 and 24 days. Thus, the operation free interval more than doubled following cidofovir use in the first patient, but was unchanged for the second.

\section{Conclusion}

A cure for RRP remains elusive. Severe RRP causes significant morbidity and mortality. Although rare, extralaryngeal disease in patients with RRP does occur and prognosis in pulmonary spread is poor. Several adjuvant therapies, such as cidofovir, have been employed. Cidofovir is a cytidine nucleoside analogue antiviral agent with broad-spectrum in vitro activity against herpesviruses, HPV, Adenovirus, polyomoviruses and human poxvirus. It is used as an intravenous treatment for CMV retinitis in AIDS patients. Efficacy has been demonstrated by clinical trials, but cross-resistance has been identified. Though data obtained from this study is inconclusive, further larger scale, long-term research is suggested.

\section{Laser surgery for laryngomalacia: the West of Scotland experience 1993-2003}

AD Whymark, WA Clements, H Kubba, NK Geddes

From the Royal Hospital for Sick Children, Yorkhill, Glasgow, UK.

\section{Introduction}

A variety of surgical techniques have been described to treat severe laryngomalacia. Division of the aryepiglottic folds is the most widely used but runs the risk of supraglottic stenosis if surgery is extensive. The standard technique used in Glasgow has been a $\mathrm{CO}_{2}$ laser supraglottoplasty, applying the laser in a linear pattern to the vallecular surface of the epiglottis, without aryepiglottic fold division. This curls the epiglottis away from the glottis preventing supraglottic collapse on inspiration. Where necessary this is augmented with laser ablation of excess mucosa over the arytenoids. We performed a retrospective audit of laser surgery performed for laryngomalacia over a ten-year period.

\section{Method}

Cases were identified by searching the hospital's theatre computer database for key words. A ten-year period was chosen and 160 case notes were identified as possibly having had surgery for laryngomalacia. These notes were searched and data collected and stored for analysis.

\section{Results}

Seventy-six children had undergone surgery for laryngomalacia between 1 January 1993 and 31 December 2002. In all children except two, the indication for surgery was stridor associated with poor feeding and failure to gain weight appropriately. One child was investigated for an 
ITU admission with croup, and one had recurrent respiratory infections.

Of these children 52 were male, 24 were female. The age range at presentation was five days to 32 months with a mean of 16 weeks. Four had neurological problems, two had a syndrome diagnosed, and two were described as dysmorphic with no syndrome diagnosed. Five had a cardiac abnormality. Seventeen were lost to follow up (11 followed up at local hospital with no further correspondence, one needed aortopexy under the cardiac surgeons, three moved out of Scotland, two did not attend follow up) with follow-up data available for 59 .

One child died of cardiac abnormalities. Eight needed a tracheostomy (six had neurological or syndromal abnormalities, and two were apparently normal children). Seven had repeat procedures to control symptoms. Five were still stridulous but weight gain was good, four had mild stridor and 34 had complete resolution of stridor. Two needed post-operative intubation of 24 hours or more, with four contracting a respiratory tract infection post operatively. There were no recorded cases of laryngeal supraglottic stenosis. Thirty-four had weight data recorded pre-operatively, at the time of operation and postoperatively. Of these 32 showed an increase in growth velocity after surgery.

\section{Conclusion}

This technique compares well to other forms of surgery for laryngomalacia. There were no serious complications of the surgery. Significant failures were common only in children with associated medical and neurological problems. Growth velocity is recommended as an objective outcome measure for laryngomalacia surgery.

\section{Laryngeal ultrasound to assess vocal fold paralysis in children}

Archana Vats, George Worley, Rose de Bruyn, Holly Porter, David Albert, Martin Bailey

From the Great Ormond Street Hospital for Sick Children, London WC1N 3JH, UK.

\section{Introduction}

Stridor in the infant and child is a difficult management issue and early, accurate elucidation of the cause is important. The current gold standard method for assessment of vocal fold movements is evaluation of the larynx by endoscopy using general anaesthesia. This method is clearly invasive and hence the desirability of a practical, reliable, non-invasive and safe method is apparent. The aim of this study was to assess the practicality and validity of laryngeal ultrasound to establish vocal fold movement in children with suspected vocal fold palsy.

\section{Method}

Fifty-five consecutive patients (age range three days to 12 years) with suspected vocal fold palsy were referred to the Department of Paediatric Otolaryngology at Great Ormond Street Hospital for Sick Children. All patients underwent both laryngeal ultrasound and rigid endoscopy under general anaesthetic, in order to allow comparison between the two modalities. Laryngeal ultrasound was performed by a single consultant radiologist. The operator visually assessed the respiratory cycle. The larynx was scanned transversely in quiet respiration and the movement of the vocal folds assessed. The position of the arytenoid cartilages was also assessed. Children were not sedated. During rigid endoscopy, vocal fold movements were assessed using the laryngoscope and telescope whilst the child was awakening from anaesthesia. All procedures were performed under direct consultant supervision. Both surgeon and radiologist were blinded to the findings of the other modality.

\section{Results}

Ultrasonographic findings correlated with endoscopic findings in 45 of the 55 patients, giving an overall concordance rate of 81.8 per cent. This, however, rose to a concordance rate of 89.5 per cent in patients aged over 12 months. Technically, unilateral palsies are considered easier to image by radiologists and this is confirmed in our study by an overall concordance rate of 88.2 per cent for unilateral vocal fold palsy compared with 82.1 per cent for bilateral vocal fold palsy.

\section{Conclusion}

Laryngeal ultrasound has been shown to be a welltolerated, safe and non-invasive technique with accuracy comparable to that of laryngoscopy, which is generally accepted to be the current gold standard. We feel that laryngeal ultrasound is a useful adjunct to endoscopy but needs to be interpreted with care in smaller children. This technical constraint may improve with the advent of new high-resolution, variable-focus, small-footprint transducers.

\section{Results of pharyngoplasty for velopharyngeal incompetence: the Birmingham Children's Hospital experience}

Chee Toh, M Siemers, J Russell, I Underwood, K Pearman From the Birmingham Children's Hospital, Birmingham B4 6NH, UK.

\section{Introduction}

Velopharyngeal incompetence (VPI) is the failure of the velopharyngeal valve to close during speech production. This results in hypernasal resonance and/or excess nasal airflow. VPI can result in cleft type speech characteristics including compensatory articulations, and at worst, unintelligible speech. Speech therapy is not usually indicated for the treatment of hypernasality due to structural abnormalities. Surgical augmentation of the palate is used to correct the area of insufficiency. Posterior pharyngeal wall flaps are widely used, with lateral and sphincter techniques also employed.

\section{Method}

We conducted a retrospective review of data on patients who had undergone pharyngoplasty from 1994 until 2003 for VPI. Sixty-three cases were identified but full pre- and post-operative data was only available for 37 patients. Data was collected on demographics, diagnosis, pre- and post-operative results, type of pharyngoplasty and major complications. Diagnosis of VPI was confirmed on lateral videofluoroscopy and by fibreoptic nasoendoscopy where appropriate. Speech assessment was conducted by specialist speech and language therapists using the GOS.SP.ASS. A superiorly based posterior pharyngeal wall flap was used for cases with larger defects and also for smaller gaps with good lateral mobility. An orticochoea lateral flap was employed where there was a coronal closure pattern and for smaller gaps where lateral mobility was poor. 


\section{Results}

Mean age was 8.1 years (range 4-16 years). Ten patients had either unilateral or bilateral cleft lip and palate, 11 patients had cleft palate alone, four patients had submucosal cleft palate and 12 patients had non-cleft VPI. Pre-operatively, 32 patients had grade 2 hypernasality and five patients had grade 1 hypernasality. Twenty-five patients underwent posterior pharyngeal wall pharyngoplasty and 12 patients underwent ortichochea pharyngoplasty. Postoperative assessments were conducted an average of 11.3 months following surgery. A total of 28 patients experienced an improvement in their GOS.SP.ASS grade for hypernasality post-operatively. Of these, 16 patients went from grade 2 hypernasality to grade 0 (normal), and four patients from grade 1 to grade 0 . Re-operation was necessary in four patients to relieve post-operative nasal obstruction (posterior technique). Two cases of postoperative bleeding required no surgical intervention.

\section{Conclusion}

Patients with cleft palate are at risk of VPI although there are patients in the non-cleft population who may also present with this difficulty. Patients requiring investigation are identified from speech assessment. Structure and function of the velopharyngeal valve is confirmed by videofluoroscopy and nasendoscopy. The surgeon selects the most appropriate operative technique in relation to the investigative findings. In this series 77.8 per cent of patients benefited from the procedure. This is consistent with the 70-90 per cent rate found in the literature. The most series complication was nasal obstruction, resulting in an 8.1 per cent re-operation rate. Hyponasality with pharyngeal flap techniques has been reported in 18 per cent of patients in other series. Pharyngoplasty for VPI is safe and effective in the majority of properly selected cases.

\section{The midfacial degloving approach to sinonasal tumours in children}

Nneka Eze, Michelle Wyatt, Dominic Bray, Martin Bailey, Benjamin Hartley

From the Great Ormond Street Hospital for Sick Children, London WC1N 3JH, UK.

\section{Introduction}

The midfacial degloving approach was originally described by Casson in 1974 but has been little used in the paediatric population. The procedure allows access to benign and malignant lesions of the sinonasal region with the avoidance of an external scar. The advantages and application of this technique are presented in nine paediatric patients. The traditional method of surgical access to the midfacial structures for the removal of benign and malignant lesions has been through external facial incisions or via the paranasal sinus or transpalatine routes. External approaches such as the Weber-Ferguson and lateral rhinotomy leave unattractive scars in the midface which carry a clear cosmetic disadvantage, particularly in the young.

\section{Method}

The midfacial degloving approach has been used in our specialist tertiary referral centre for the treatment of nine children in the last two years. The technique has been utilised for both benign and malignant conditions affecting the nose, paranasal sinuses, and nasopharynx. A retrospective case notes review was performed on these children. Demographic data, indications, results and complications were noted.

\section{Results}

For the past two years this technique has been used in nine children ranging in age from three months to 15 years (mean age five years). The male to female ratio was 5:4. Follow-up was from one month to two years (mean seven months). There were no complications encountered during this period of follow-up. No recurrence has yet been identified and revision surgery was not required in any of the cases. In all cases adequate surgical exposure was achieved and surgical excision was macroscopically complete. In no case was an additional external incision required to complete the procedure.

\section{Conclusion}

There is limited data on the use of this approach in children as concerns have arisen regarding the effects of radical surgery on midfacial development. No cosmetic deformities occurred relating to midfacial development in this series during our limited period of follow-up. Our results concur with those produced by Conley et al. ${ }^{1}$ who followed up babies and children for 10 years and demonstrated no facial growth disturbances. There has been anxiety regarding the effect of radical surgery on midfacial development in children under the age of two years. These concerns have been deemed to be unfounded. Results from continuing studies assessing standardised sequential photographs and lateral cephalometry on children undergoing radical sinonasal surgery with various approaches, show no evidence of abnormal midfacial growth particularly if the hard palate, cartilaginous septum and upper lateral cartilages are undisturbed.

\section{Reference \\ 1 Conley J, Price J. Sublabial approach to the nasal and nasopharyngeal cavities. Am J Surg 1979;138:615-8}

\section{Consent issues - does the practice follow General Medical Council guidelines?}

M K Aneeshkumar, E Z Osman, R W Clarke

From the Alder Hey Hospital, Eaton Road, Liverpool, UK.

\section{Introduction}

Through the booklet Seeking patients' consent: the ethical consideration, the General Medical Council (GMC) sets out the principles of good practice which all registered doctors are expected to follow when seeking patients' informed consent. The methods of taking consent for surgery should be uniform throughout the country. With the present culture of litigation, it is important to have standardization. To analyse this we conducted a questionnaire survey.

\section{Method \\ Questionnaires were sent to 200 ENT consultants and 200 ENT trainees. Seventy-nine (39.5 per cent) of consultants and 85 (43 per cent) of trainees responded. Responses were analysed.}

\section{Results}

The trainees' perspective of who takes consent differed from the consultants. According to the consultants, the majority of the consents were taken by the consultant themselves (30 per cent); senior house officer ( 25 per cent) 
and registrars ( 6 per cent). But according to the trainees, consent was mainly taken by senior house officer (36 per cent), registrars (20 per cent) and consultants ( 5 per cent). In their department, only 11 per cent of the consultants felt that the person taking consent was not competent to perform the operation and they are all trained to take consent if not competent to do the procedure. Thirty-eight per cent of the trainees felt they are taking consent for surgeries, which they are not competent to do, and 16 per cent of these felt they are not even trained to take consent. Forty-nine per cent of the trainees and 61 per cent of the consultants provided patient information leaflets.

Regarding parental responsibility, 20 per cent of the trainees and consultants specifically ask the mother to sign the consent form for a child's operation. The majority of trainees and consultants do not ask the marital status of the father signing the form. Fifty-two per cent of trainees and 52 per cent of consultants will not take consent if they knew the father was not married. Thirty-one per cent of trainees and 35 per cent of consultants do not use the new consent form, which provides a copy for the patient.

\section{Conclusion}

There is no uniformity on different issues related to consent in following GMC guidelines. There is significant difference in the opinion of trainees and consultants about the competence of the person taking consent for major surgeries. The new consent form is not implemented through out the country. The importance of parental responsibility is considered differently among trainees and consultants. All these disparities can cause problems when litigation arises and also when the trainee rotates to different hospitals.

\section{Changing practice in paediatric ENT in the last decade}

Alok Sharma, Andrew Bath

From the Norfolk \& Norwich University Hospital, Norwich NR4 7UY, UK.

\section{Introduction}

Over the last decade, advances in clinical governance and evidence-based medicine have had a significant impact in many areas of medicine. The aim of this study was to investigate whether this had affected paediatric ENT surgical practice.

\section{Method \\ Computerised theatre records of the Norfolk and Norwich University Hospital were retrospectively reviewed from 1 January 1994 to 31 December 2003. The numbers of paediatric ENT operations (adenoidectomy, tonsillectomy and grommet insertion) performed on an annual basis was calculated.}

\section{Results}

In 2003, the numbers of tonsillectomy (187) and adenoidectomy (130) procedures performed were less than one third of the numbers performed in 1994 (588 and 417 respectively); and grommet insertions had fallen in 2003 (363) to less than two thirds of that in 1994 (544).

\section{Discussion}

Over this 10-year period the numbers of basic paediatric ENT operations performed have steadily fallen, without major changes in environment, society or the population of Norfolk. The reasons for this are unclear.
The decision for surgery is dependent upon the combined agreement of the doctor and patient/parent. From the doctor's perspective they have become more 'accountable' due to national prospective audits of clinical practice that have led to the introduction of guidelines for surgery e.g. The TARGET study and the Scottish National Tonsillectomy Audit. From the patient's/parent's perspective they are better informed regarding medical treatment and its associated risks e.g. vCJD. These and other factors may have led to a more cautious approach to surgery in this population.

\section{Conclusion}

There has been a dramatic change in paediatric ENT surgical practice over the last decade. The value of these operations has been proven by large national studies. Have we become too cautious?

\section{The future provision of paediatric otolaryngology training}

Charlie Hall, Mike Saunders, Kate Evans, Adrian James From the Bristol Royal Hospital for Children, Bristol BS2 8BJ, UK.

\section{Introduction}

Specialist training in otolaryngology has significantly altered in the last ten years initially through changes introduced by Calman, and now under the influence of the European Working Time Directive and Modernising Medical Careers. Paediatric training has been further influenced by moves towards centralisation and alteration in anaesthetic guidelines for treating children. We present a current perspective of specialist registrar $(\mathrm{SpR})$ training in paediatric otolaryngology through a questionnairebased survey of paediatric experience over a one-year period among SpRs in the South West (SW) England. We examine how future training may be affected by planned changes in the SpR training structure.

\section{Method}

An e-mail questionnaire was distributed to all SpRs in SW England to evaluate their paediatric experience over a one-year period. Trainees who had spent part of the year away from the rotation (e.g. on fellowships abroad) or did not complete a full year's training at registrar level were excluded. Four operations (paediatric tracheostomy $[<2$ years], paediatric microlaryngobronchoscopy [MLB], pinnaplasty and choanal atresia repair) were identified, and trainees asked to extract from their surgical log-books the number of these procedures that they had experienced over a one-year period (October 2002 to October 2003). Further questions were asked to evaluate: attendance at paediatric resuscitation courses, training in communication skills and experience from specialist paediatric clinics.

\section{Results}

Questionnaires were sent to 14 eligible SpRs with a 100 per cent response rate. 80 per cent (8/10) of infant tracheostomies were experienced in the tertiary referral centre, the remainder occurring in one of the other teaching hospitals in the region. Forty-five per cent (39/83) of paediatric MLBs took place at the tertiary referral centre with a further 37 per cent (17/83) amongst the other teaching hospitals. The majority (64 per cent, 16/25) of therapeutic MLBs were experienced at the tertiary referral centre. Experience of MLBs for foreign body removal was fairly evenly spread across the region. The 
majority (70 per cent, 7/10) of choanal atresia repairs occurred at the tertiary referral centre. Experience of pinnaplasty and attendance at special paediatric clinics was evenly distributed across the region.

\section{Conclusions}

The proposed reduction in length of $\mathrm{SpR}$ training and increases in the number of trainees may reduce the availability of sub-specialist attachments within a regional training rotation. Valuable paediatric experience is available across the SW region, particularly from the teaching hospitals. However, for most procedures, the majority of paediatric experience is gained at the tertiary referral centre. This must be considered in the organisation of $\mathrm{SpR}$ rotations, especially for the provision of 'emergency safe' training in the management of the paediatric airway. 